\title{
Simplified Discontinuous Galerkin Methods for Systems of Conservation Laws with Convex Extension
}

\author{
Timothy J. Barth \\ NASA Ames Researih Center, Information Sciences Directorate. Moffett Field, \\ C.t 94035 . LSA \\ barth $q_{\text {nas.nasa.gov }}$
}

\begin{abstract}
Simplified forms of the space-time discontinuous Galerkin (DG) and discontinuous Galerkin least-squares (DGLS) finite element method are developed and analvzed. The new formulations exploit simplifying properties of entropy endowed conservation law systems while retaining the favorable energy properties associated with symmetric variable formulations.
\end{abstract}

\section{Introduction}

The high-order accurate numerical solution of systems of nonlinear conservation laws remains a computationally expensive endeavor. This article considers simplified forms of the Discontinuous Galerkin (DG) and Discontinuous Galerkin least-squares (DGLS) finite element methods tailored to systems of first-order nonlinear conservation laws with convex entropy extension. Central to the development is the Eigenvalue Scaling Theorem which characterizes right symmetrizers of entropy endowed systems of conservation laws in terms of scaled eigenvectors of the corresponding flux Jacobian matrices. This yields a simplification of the DG and DGLS methods without sacrificing the pleasing energy properties of symmetric variable formulations. The next section briefly reviews a number of results in symmetrization theory discussed in detail in Barth [2.1].

\section{Brief Review of Symmetrization and the Eigenvector Scaling Theorem}

Consider a system of $m$ coupled first-order differential equations in $d$ space coordinates and time which represents a conservation law process. Let $u(x, t)$ : $\mathbb{R}^{d} \times \mathbb{R}^{+} \mapsto \mathbb{R}^{m}$ denote the dependent solution variables and $\boldsymbol{f}(\boldsymbol{u}): \mathbb{R}^{m} \mapsto$ $\mathbb{R}^{m \times d}$ the flux vector. The prototype conservation system is then given by

$$
u_{. t}+f_{. x_{i}}^{i}=0
$$

with implied summation on the index $i$. Additionally. the system is assumed to possess an scalar entropy extension. Let $U(\boldsymbol{u}): \mathbb{R}^{m} \mapsto \mathbb{R}$ denote an 
entropy function and $F(\boldsymbol{u}): \mathbb{R}^{m} \mapsto \mathbb{R}^{d}$ the entropy flux such that in addition to (1.1) the following inequality holds

$$
\tau_{. t}+F_{x_{i}}^{i} \leq 0
$$

with equality for smooth solutions. In symmetrization theory for first-order conservation laws, one seeks a mapping $\boldsymbol{u}(\boldsymbol{v}): \mathbb{R}^{m} \mapsto \mathbb{R}^{m}$ applied to (1.1) so that when transformed

$$
u_{. v} v_{. t}+f_{, v}^{i} v_{, x_{i}}=0
$$

the matrix $u_{. v}$ is symmetric positive definite (SPD) and the matrices $f_{.}^{i}$ are symmetric. Clearly, if functions $\mathcal{U}(\boldsymbol{v}), \mathcal{F}^{i}(\boldsymbol{v}): \mathbb{R}^{m} \rightarrow \mathbb{R}$ can be found so that

$$
u^{T}=\mathcal{U} . v, \quad\left(f^{i}\right)^{T}=\mathcal{F}_{, v}^{i}
$$

then the matrices

$$
\boldsymbol{u}_{. v}=\mathcal{U}_{, v, v}, \quad f_{, v}^{i}=\mathcal{F}_{, v, v}^{i}
$$

are symmetric. Since $v$ is not yet known, little progress has been made. Introducing the following duality relationships

$$
\begin{gathered}
U(u)=v^{T}(u) u-\mathcal{U}(v(u)) \\
F^{i}(u)=v^{T}(u) f^{i}(u)-\mathcal{F}^{i}(v(u))
\end{gathered}
$$

followed by differentiation yields

$$
\begin{gathered}
U_{, u}=v^{T}+u^{T} v_{, u}-\mathcal{U}_{, v} v_{, u}=v^{T} \\
F_{, u}^{i}=v^{T} f_{, u}^{i}+\left(f^{i}\right)^{T} v_{, u}-\mathcal{F}_{, v}^{i} v_{, u}=v^{T} f_{, u}^{i}
\end{gathered}
$$

Equation (1.8) gives an explicit expression for the entropy variables $v$ in terms of derivatives of the entropy function $U(\boldsymbol{u})$

$$
v^{T}=U_{, u}
$$

Finally, we require convexity of $U(\boldsymbol{u})$ which insures positive definiteness of $v_{, u}$ and $u_{, v}$ and implies hyperbolicity of $(1.1)[5,9]$, viz., that the linear combination $f_{, u}(n)=n_{i} f_{, u}^{i}$ has real eigenvalues and a complete set of realvalued eigenvectors for all nonzero $n \in \mathbb{R}^{d}$. This result follows immediately from the identity

$$
\left(u_{. v}\right)^{-1 / 2} f_{. u}(n)\left(u_{, v}\right)^{1 / 2}=\underbrace{\left(u_{, v}\right)^{-1 / 2} f_{, v}(n)\left(u_{, v}\right)^{-1 / 2}}_{\text {symm }}
$$

which shows that $f_{. u}(n)$ is similar to a symmetric matrix. 


\subsection{The Eigenvector Scaling Theorem}

Next, we consider an important algebraic property of right symmetrizable sistems which is used later in the implementation of the DG and DGLS shemes. Simplifying upon the previous notation. let $\tilde{I}_{0}=u_{v} . A_{2}=\boldsymbol{f}_{v}^{\prime}$, $\dot{I}_{1}=A_{i} \tilde{t}_{0}$ and rewrite (1.3)

$$
\tilde{A}_{0} v_{. t}+\tilde{A}_{i} v_{. x_{i}}=0
$$

The following theorem states a property of the symmetric matrix $\tilde{A}_{i}$ symmetrized via the symmetric positive definite matrix $\bar{t}_{0}$.

Theorem 1.1 (Eigenvector Scaling). Let $A \in \mathbb{R}^{n \times n}$ be an arbitrary diagonalizable matrix and $S$ the set of all right symmetrizers:

$$
S=\left\{B \in \mathbb{R}^{n \times n} \mid B \text { SPD, } A B \text { symmetric }\right\} \text {. }
$$

Further. let $R \in \mathbb{R}^{n \cdot n}$ denote the right eigenvector matrix which diagonalizes A

$$
A=R \cdot 1 R^{-1}
$$

with $r$ distinct eigenvalues, $.1=\operatorname{Diag}\left(\lambda_{1} I_{m_{1} \times m_{1}}, \lambda_{2} I_{m_{2} \times m_{2}} \ldots, \lambda_{r} I_{m_{r} \times m_{r}}\right)$. Then for each $B \in S$ there exists a symmetric block diagonal matrix $T=$ $\operatorname{Diag}\left(T_{m_{1} \times m_{1}}, T_{m_{2} \times m_{2}} \ldots, T_{m_{+} \times m_{r}}\right)$ that block scales columns of $R, \tilde{R}=$ $R T$. such that

$$
B=\tilde{R} \tilde{R}^{T} . \quad A=\tilde{R} \cdot 1 \tilde{R}^{-1}
$$

which imply that

$$
A B=\tilde{R} \cdot 1 \tilde{R}^{T}
$$

Proof. Omitted, see [2].

Note that this last formula states a congruence relationship since $\tilde{R}$ is not generally orthonormal and $A$ does not represent the eigenvalues of $A B$. The Eigenvalue Scaling Theorem is a variant of the well known theory developed for the commuting matrix equation $A X-X A=0, A, X \in \mathbb{R}^{n \times n}$, see for example Gantmacher [6]. Examples of the Eigenvector Scaling Theorem for the Euler and magnetohydrodynamic equations are given in [2].

\subsection{Generalized Matrix Functions with Respect to the Riemannian Matrix $\overline{\boldsymbol{A}}_{0}$}

For use in later developments, it is useful to define the following generic matrix function $f(\tilde{A})$ with respect to the Riemannian matrix $\tilde{A}_{0}$

$$
f_{\tilde{A}_{0}}(\tilde{A}) \equiv \tilde{I}_{0} f\left(\tilde{A}_{0}^{-1} \tilde{A}\right)
$$

This definition reflects the following steps: (1) multiplication of the system (1.11) by $\bar{A}_{0}^{-1}$ in order to restore a Euclidean metric, (2) invocation of the 
matrix function on the matrix product $\bar{A}_{0}^{-1} \bar{A}_{i},(3)$ multiplication of the result by $\overline{\mathcal{A}}_{0}$ to restore the original metric matrix. Proposition 1.2 shows that this generalized matrix finction is symmetric and has a rather simple construction for symmetrizable s*stems by exploiting the Eigenvalue Scaling Theorem.

Proposition 1.2. Burth [2,1]. Let $\bar{A}_{0}$ denote the SPD right symmetrizer of $A$ such that $\bar{A}=A . \bar{I}$. $\bar{I}_{0}=\bar{R} \tilde{R}^{T}$. und $A=\tilde{R} \cdot 1 \bar{R}^{-1}$. The generalized matrix function $f_{.,}(. \bar{j})$ is ,ymmetric and defined canonically in terms of entropy scaled eigeniectors is

$$
f_{\tilde{i}_{0}}(\tilde{A})=\tilde{R} f(\Lambda) \dot{R}^{T}
$$

In later sections, the generalized matrix absolute value function $|\bar{i}| \bar{t}_{0}$ will be required

$$
|\tilde{A}|_{., 1}=\tilde{R}|\cdot 1| \tilde{R}^{T}
$$

This matrix absolute value function has a natural generalization to $\mathbb{R}^{d}$ using an $L_{p}$-like norm defnition

$$
|\tilde{A}|_{p, \bar{A}_{0}}=\left(\sum_{i=1}^{d}\left|\mathcal{H}_{i}\right|^{p}\right)^{1 / p} \tilde{A}_{0}
$$

which has a particularly simple form when $p=1$ which is used later in the least-squares term appearing in the DGLS method

$$
|\bar{A}|_{1 . . \bar{t}_{0}}=\sum_{i=1}^{d} \tilde{R}_{i}\left|1_{i}\right| \bar{R}_{i}^{T} \text {. }
$$

\section{Simplified DG, DGLS, and GLS Finite Element Methods}

Let $\Omega$ denote a spatial domain composed of nonoverlapping elements $T_{i}$, $\Omega=\cup T_{i}, T_{i} \cap T_{j}=\emptyset, i \neq j$ and $\left.I^{n}=\right] t^{n}, t^{n+1}$ [ the $n$-th time interval. It is useful to also define the element set $\mathcal{T}=\left\{T_{1}, T_{2}, \ldots, T_{|\mathcal{T}|}\right\}$ and edge set $\mathcal{E}=\left\{e_{1}, e_{2}, \ldots e_{E}\right\}$. To simplify the exposition, consider a single variational formulation with weakly enforced boundary conditions. By choosing the correct space of functions (discontinuous or continuous) and omitting the least-squares variational term, one can switch from the DGLS formulation to the DG or GLS formulations. In the GLS formulation $[7,10]$, functions are continuous in space and discontinuous in time

$$
\mathcal{V}^{h}=\left\{v^{h} \mid \boldsymbol{v}^{h} \in\left(C^{0}\left(\Omega \times I^{n}\right)\right)^{m}, \boldsymbol{v}_{\left.\right|_{T \times I^{n}}}^{h} \in\left(\mathcal{P}_{k}\left(T \times I^{n}\right)\right)^{m}\right\}
$$

where $v$ denotes the entropy variables for the system. In the DG and DGLS formulations [8,3], functions are discontinuous in space and time, i.e.

$$
\mathcal{L}^{\cdot h}=\left\{\boldsymbol{v}^{h} \mid \boldsymbol{v}_{\left.\right|_{T \times I^{n}} ^{h}} \in\left(\mathcal{P}_{k}\left(T \times I^{n}\right)\right)^{m}\right\} \text {. }
$$


Consider the prototype hyperbolic system for the space-time domain $\Omega \times[0, T]$ with boundary data $g$ imposed on $\Gamma$ via admissibility condition

$$
\begin{array}{rlrl}
\boldsymbol{u}_{. t}+\boldsymbol{f}_{. x_{t}}^{\imath} & =0 & \text { in } \Omega \\
\mathcal{A}^{-}(\boldsymbol{n})(\boldsymbol{g}-\boldsymbol{u}) & =0 & & \text { on } \Gamma
\end{array}
$$

or in symmetric quasi-linear form for smooth solutions

$$
\begin{array}{ll}
\bar{A}_{0} v_{. t}+\tilde{I}_{,} v_{x_{1}}=0 & \text { in } \Omega \\
\tilde{I}^{-}(\boldsymbol{n})(\bar{g}-\boldsymbol{v})=0 & \text { on } \Gamma
\end{array}
$$

with $\mathcal{A}(\boldsymbol{n})=\boldsymbol{n}_{i} \mathcal{A}_{i}$ and $\bar{I}(\boldsymbol{n})=\boldsymbol{n}_{i} \dot{I}_{i}$. The combined GLS and DG schemes are defined by the following stabilized variational formulation:

Find $v^{h} \in \mathcal{V}^{\text {h }}$ such that for all $\boldsymbol{w}^{h} \in \mathcal{L}^{\text {h }}$

$$
\begin{aligned}
& B\left(\boldsymbol{v}^{h} \cdot u^{h}\right)_{\text {(iAL }}+B\left(\boldsymbol{v}^{h}, \boldsymbol{w}^{h}\right)_{\mathrm{LS}}+B\left(\boldsymbol{v}^{h}, \boldsymbol{w}^{h}\right)_{\mathrm{BC}}=0 \\
& B(\boldsymbol{v} \cdot \boldsymbol{w})_{(;+\mathrm{L}}=\int_{I^{n}} \int_{\Omega}\left(-\boldsymbol{u}(v) \cdot \boldsymbol{w}_{. t}-f^{i}(v) \cdot w_{x_{i}}\right) d x d t \\
& +\int_{\Omega}\left(\boldsymbol{w}\left(t_{-}^{n+1}\right) \cdot \boldsymbol{u}\left(\boldsymbol{v}\left(t_{-}^{n+1}\right)\right)-\boldsymbol{w}\left(t_{+}^{n}\right) \cdot \boldsymbol{u}\left(\boldsymbol{v}\left(t_{-}^{n}\right)\right)\right) d x \\
& +\int_{I^{n}} \sum_{\varepsilon=\varepsilon^{-}} \int_{e}\left(w\left(x_{-}\right)-\boldsymbol{w}\left(x_{+}\right)\right) \cdot \boldsymbol{h}\left(\boldsymbol{v}\left(x_{-}\right), \boldsymbol{v}\left(x_{+}\right) ; \boldsymbol{n}\right) d x d t \\
& B\left(\boldsymbol{v} \cdot \boldsymbol{u}: \mathrm{Ls}=\int_{I^{n}} \sum_{T \in \mathcal{T}} \int_{T}\left(\tilde{I}_{0} \boldsymbol{w}_{. t}+\tilde{A}_{i} \boldsymbol{w}_{x_{1}}\right) \cdot \boldsymbol{\tau}\left(\tilde{A}_{0} \boldsymbol{v}_{. t}+\tilde{A}_{i} \boldsymbol{v}_{x_{i}}\right) d x d t\right. \\
& B(\boldsymbol{v}, \boldsymbol{w})_{\mathrm{BC}}=\int_{I^{n}} \int_{\Gamma} \boldsymbol{w} \cdot \boldsymbol{h}(\boldsymbol{v}, \tilde{\boldsymbol{g}} ; \boldsymbol{n}) d x d t
\end{aligned}
$$

where $h$ denotes a numerical flux function and $\tau$ a small $m \times m$ SPD matrix for the least-squares term. For theoretical and practical reasons, two numerical flux functions are considered. Both are of the form

$$
\boldsymbol{h}\left(\boldsymbol{v}_{-}, \boldsymbol{v}_{+} ; \boldsymbol{n}\right)=\frac{1}{2}\left(\boldsymbol{f}\left(\boldsymbol{v}_{-} ; \boldsymbol{n}\right)+\boldsymbol{f}\left(\boldsymbol{v}_{+} ; n\right)\right)-\frac{1}{2} \boldsymbol{h}^{d}\left(\boldsymbol{v}_{-}, \boldsymbol{v}_{+} ; \boldsymbol{n}\right)
$$

and consistent with the true flux in the sense that $h(v, v ; n)=f(v ; n)$.

1. Symmetric Mean-Value Flux. This flux is motivated from the nonlinear energy theory of Sect. 2.2. Define the parameterization $\overline{\boldsymbol{v}}(\theta) \equiv$ $v\left(x_{-}\right)+\theta[v]_{x_{-}}^{x_{+}}$. The symmetric mean-value flux is then given by

$$
h_{\text {SMV }}^{d}\left(v_{-}, v_{+} ; n\right)=\left|\tilde{A}\left(v_{-}, v_{+} ; n\right)\right|_{\text {SMV }}[v]_{x_{-}}^{x_{+}}
$$

with

$$
\left|\overline{\mathcal{I}}\left(\boldsymbol{v}_{-}, \boldsymbol{v}_{+} \bar{n} \boldsymbol{n}\right)\right|_{\mathrm{S} M V} \equiv \int_{0}^{1}|\overline{\mathcal{I}}(\overline{\boldsymbol{v}}(\theta) ; \boldsymbol{n})|_{\bar{A}_{0}} d \theta
$$

By construction. the matrix $\left|\overline{\mathcal{A}}\left(\boldsymbol{v}_{-}, \boldsymbol{v}_{+} ; \boldsymbol{n}\right)\right|_{\mathrm{S} M \mathrm{~V}}$ is symmetric positive semi-definite. Lsing this form of flux dissipation (2.5), nonlinear entropy 
norm stability of the DG, DGLS, and GLS formulations can be shown as discussed in Sect. 2.2. In addition, let

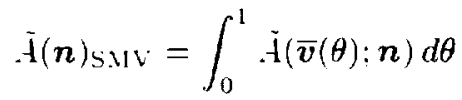

denote the integ:al mean-value matrix for which the following useful property exists

$$
[f(n)]_{x_{-}}^{x_{+}}=\tilde{I}(n)_{\text {SMV }}[v]_{x_{-}}^{x_{+}}
$$

which is a necessary ingredient for optimal discontinuity resolution. To prove stability of other (more practical) forms of flux dissipation, one formally needs only show that the new form is more energy dissipative than the symmetric mean-value form in the following sense:

$$
[\boldsymbol{v}]_{x_{-}}^{x_{+}} \cdot \boldsymbol{h}_{\mathrm{SMV}}^{d} \leq[\boldsymbol{v}]_{x_{-}}^{x_{+}} \cdot \boldsymbol{h}^{d}
$$

2. Discrete Symmetric Mean-Value Flux. The discrete symmetric meanvalue flux function replaces the state-space path integration in (2.5) by $\therefore$ point numerical quadrature

$$
\boldsymbol{h}_{\mathrm{DSMV}}^{d}\left(\boldsymbol{v}_{-}, \boldsymbol{v}_{+} ; \boldsymbol{n}\right)=\left|\tilde{\mathrm{A}}\left(\boldsymbol{v}_{-}, \boldsymbol{v}_{+} ; \boldsymbol{n}\right)\right|_{\mathrm{DSMV}}[\boldsymbol{v}]_{x_{-}}^{x_{+}}
$$

with

$$
\left|\bar{A}\left(\boldsymbol{v}_{-}, \boldsymbol{v}_{-}: \boldsymbol{n}\right)\right|_{\mathrm{DSMV}}[\boldsymbol{v}]_{x_{-}}^{\boldsymbol{x}_{+}} \equiv \sum_{q=1}^{N} w_{q}\left|\tilde{A}\left(\bar{v}\left(\theta_{q}\right) ; \boldsymbol{n}\right)\right|_{\tilde{A}_{0}}[\boldsymbol{v}]_{x_{-}}^{x_{+}}
$$

where $u_{q}$ and $\theta_{q}$ denotes the quadrature weights and positions. In forming this flux, recall from the Eigenvalue Scaling Theorem that $\mid \tilde{A}_{\tilde{A}_{0}}=$ $\bar{R}|i| \mid \tilde{R}^{T}$. This flux function is of practical interest since it is easily formed and has a relatively straightforward Jacobian linearization as will be shown later. The absolute value in this equation renders the state-space path integration from $v_{-}$to $v_{+}$slope discontinuous whenever entries in $A$ change sign. In this case, to retain accuracy of the numerical quadrature at $n \leq m$ possible points of slope discontinuity, the path integration is further subdivided into subintervals, e.g. $\left[\boldsymbol{v}_{-}, \boldsymbol{v}_{+}\right]=\left[\boldsymbol{v}_{-}, \boldsymbol{v}_{\mathbf{1}}^{*}\right] \cup\left[\boldsymbol{v}_{\mathbf{i}}^{*}, \boldsymbol{v}_{2}^{*}\right] \cup$ $\ldots \cup\left[v_{n}, v_{+}\right]$where $v_{i}^{*} \equiv v\left(\theta_{i}\right)$ is a location $\theta_{i}$ such that an entry of .1 vanishes. In practice, satisfactory results [1] have been obtained using 2 -point Gaussian quadrature rules (which integrate cubic polynomials exactly).

\subsection{Linear Energy Analysis}

Due to length constraints of this article, we simply restate some relevant theorems given in Barth $[2,1]$ concerning energy boundedness of variational form (2.3) for systems of hyperbolic equations. 
Theorem 2.1. Global Energy Stability (Linear Hyperbolic System). The variational formulation (2.3) for linear hyperbolic systems is energy stable (modulo data $\dot{\boldsymbol{g}}$ ) with the following global energy balance:

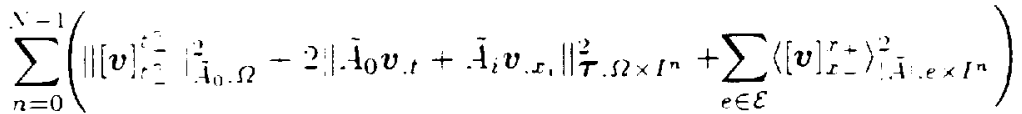

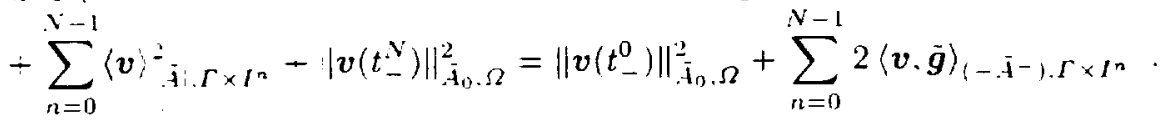

Proof. Omitted, see 2.1].

This energ: balance equation formally bounds the energy at time $t_{-}^{N}$ in terms of initial daia and inflow boundary data. Next, we consider the full nonlinear system of conservation laws.

\subsection{Nonlinear Energy Analysis}

Before presenting the nonlinear energy result, we prove a general lemma and consequential corollaries concerning entropy function/flux jump identities at space-time slab interfaces. Note that throughout this section, we utilize the state-space parameterization

$$
\bar{v}(\theta) \equiv \boldsymbol{v}\left(x_{-}\right)+\theta[\boldsymbol{v}]_{x_{-}}^{x_{+}}
$$

(similarly across time slab interfaces) for use in state-space path integrations and the interface averaging operator

$$
\langle\langle v\rangle\rangle_{x_{-}}^{x_{+}} \equiv \frac{v\left(x_{-}\right)+v\left(x_{+}\right)}{2} .
$$

Lemma 2.2. Interface Jump Identities. Let $Z(u), \mathcal{Z}(v): \mathbb{R}^{m} \mapsto \mathbb{R}$ be twice differentiable functions of their argument satisfying the duality relationship

$$
Z(\boldsymbol{u})+\mathcal{Z}(\boldsymbol{v})=\mathcal{Z}_{. v} v
$$

The following jump identities hold across interfaces

$$
\begin{array}{r}
{[Z]_{x_{-}}^{x_{+}-}\left[\mathcal{Z}_{v_{-}}\right]_{x_{-}}^{x_{+}} v\left(x_{-}\right)+\int_{0}^{1}(1-\theta)[v]_{x_{-}}^{x_{+}} \cdot \mathcal{Z}_{, v, v}(\bar{v}(\theta))[v]_{x_{-}}^{x_{+}} d \theta=0} \\
{[Z]_{x_{-}}^{x_{+}}-\left[\mathcal{Z}_{, v}\right]_{x_{-}}^{x_{+}} \boldsymbol{v}\left(x_{-}\right)-\int_{0}^{1} \theta[\boldsymbol{v}]_{x_{-}}^{x_{+}} \cdot \mathcal{Z}_{, v, v}(\bar{v}(\theta))[v]_{x_{-}}^{x_{+}} d \theta=0 .}
\end{array}
$$

Proof. Recall the following forms of Taylor series with integral remainder

$$
\begin{array}{r}
{[\mathcal{Z}]_{x_{-}}^{x_{-}}-\mathcal{Z}_{. v}\left(x_{-}\right)[v]_{x_{-}}^{x_{+}}+\int_{0}^{1} \theta[v]_{x_{-}}^{x_{+}} \cdot \mathcal{Z}_{. v \cdot v}(\bar{v}(\theta))[v]_{x_{-}}^{x_{+}} d \theta=0} \\
{[\mathcal{Z}]_{x_{-}}^{x_{+}}-\mathcal{Z}_{. v}\left(x_{-}\right)[v]_{x_{-}}^{x_{-}}-\int_{0}^{1}(1-\theta)[v]_{x_{-}}^{x_{+}} \cdot \mathcal{Z}_{. v \cdot v}(\bar{v}(\theta))[v]_{x_{-}}^{x_{+}} d \theta=0}
\end{array}
$$


and the jump form of $(2.9)$

$$
[\mathcal{Z}]_{x_{-}}^{x_{+}}-[Z]_{x_{-}}^{x_{+}}=\left\langle\left\langle\mathcal{Z}_{. v}\right\rangle\right\rangle_{x_{-}}^{x_{+}}[v]_{x_{-}}^{x_{+}}+\langle\langle v\rangle\rangle_{x_{-}}^{x_{+}}\left[\mathcal{Z}_{.}\right]_{x_{-}}^{x_{+}} .
$$

('ombining $2.11 \mathrm{a}) .2 .11 \mathrm{~b})$ and (2.12) vields

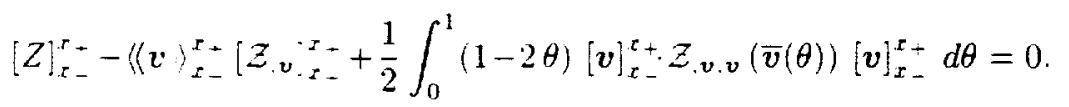

Finally: algebraically manipulating this form together with the mean-value identity

$$
\left.\mathcal{Z}_{. v}\right]_{x_{-}}^{x_{+}}=\int_{0}^{1} \mathcal{Z}_{. v, v}(\bar{v}(\theta))[\boldsymbol{v}]_{x_{-}}^{x_{+}} d \theta
$$

produces the stated lemma.

Corollary 2.3. Temporal Space-Time Slab Interface Identity. Let $t_{ \pm}$ denote a temporal space-time slab interface. The following entropy function jump identity holds across time slab interfaces

$$
\int_{\Omega}\left(\left[C^{-}\right]_{t_{-}}^{t_{-}}-v^{T}\left(t_{+}\right)[u]_{t_{-}}^{t_{+}}\right) d x+\frac{1}{2} \|\left[[\boldsymbol{v}]_{t_{-}^{+}}^{t_{+}}\|\|_{\tilde{A}_{0} \Omega}^{2}=0\right.
$$

where

$$
\|[\boldsymbol{v}]_{t_{-}}^{t_{-}}{\overline{I_{0} . \Omega}}_{\Omega} \equiv \int_{\Omega} \int_{0}^{1} 2(1-\theta)[\boldsymbol{v}]_{t_{-}}^{t_{+}} \cdot \tilde{t}_{0}(\widetilde{\boldsymbol{v}}(\theta))[\boldsymbol{v}]_{t_{-}}^{t_{+}} d \theta d x \geq 0 .
$$

Proof. Set $Z=t^{*} . Z=U$ with $\mathcal{U}_{. v}=u^{T}$ and $\mathcal{U}_{, v, v}=\bar{I}_{0}$ in Lemma 2.2 and replace $x_{ \pm}$with $t_{ \pm}$in $(2.10 \mathrm{a})$, see [10] for an alternative form.

Corollary 2.4. Spatial Space-Time Slab Interface Identity. Let $x_{ \pm}$ denote a spatial element interface. The following entropy flux jump identity holds across spatial element interfaces

$$
\left[F^{i}\right]_{x_{-}}^{x_{+}}-\left\langle\left\langle v^{T}\right\rangle\right\rangle_{x_{-}}^{x_{+}}\left[f^{i}\right]_{x_{-}}^{x_{+}}+\frac{1}{2} \int_{0}^{1}(1-2 \theta)[v]_{x_{-}}^{x_{+}} \cdot \tilde{A}_{i}(\bar{v}(\theta))[v]_{x_{-}}^{x_{+}} d \theta=0 .
$$

Proof. Set $Z=F^{i} . \mathcal{Z}=\mathcal{F}^{i}, i=1, \ldots, d$ with $\mathcal{F}_{, v}^{i}=\left(f^{i}\right)^{T}$ and $\mathcal{F}_{, v, v}^{i}=\tilde{A}_{i}$ in (2.13) of Lemma 2.2.

Note that in actual numerical calculations, it is desirable to use the variational form given by (2.3) since integration by parts has been used to insure exact discrete conservation even with inexact numerical quadrature of the various integrals. For analysis purposes, however, it is desirable to use the following equivalent non-integrated-by=parts formulation:

Find $v^{h} \in L^{h}$ such that for all $w^{h} \in \nu^{h}$

$$
B\left(\boldsymbol{v}^{h} \cdot \boldsymbol{w}^{h}\right)_{\mathrm{GAL}}+B\left(\boldsymbol{v}^{h} \cdot \boldsymbol{w}^{h}\right)_{\mathrm{LS}}+B\left(\boldsymbol{v}^{h}, \boldsymbol{w}^{h}\right)_{\mathrm{BC}}=0
$$




$$
\begin{aligned}
& B(\boldsymbol{v}, \boldsymbol{w})_{\mathrm{GAL}}=\int_{\Gamma^{1}} \int_{\Omega} \boldsymbol{w} \cdot\left(\boldsymbol{u} . t+f_{. x_{\mathrm{i}}}^{i}(\boldsymbol{v})\right) d x d t \\
& +\int_{0} \boldsymbol{w}\left(t_{+}^{n}\right) \cdot[\boldsymbol{u}]_{t_{+}^{n_{+}}}^{t_{+}^{n}} d x \\
& +\int_{I^{-}} \sum_{t \in \mathcal{E}} \int_{e} \frac{1}{2}[\boldsymbol{w}]_{x_{-}}^{x_{+}} \cdot \boldsymbol{h}^{d t}\left(\boldsymbol{v}\left(x_{-}\right) \cdot \boldsymbol{v}\left(x_{+}\right): n\right) d x d t \\
& +\int_{I^{n}} \sum_{-=\varepsilon} \int_{e}\left\langle\langle\boldsymbol{w}\rangle_{x_{-}}^{x_{+}} \cdot[\boldsymbol{f}(\boldsymbol{v} ; \boldsymbol{n})]_{x_{-}}^{x_{+}} d x d t\right. \\
& B(\boldsymbol{v} \cdot \boldsymbol{w})_{\mathrm{LS}}=\int_{T^{n}} \sum_{T \in \mathcal{T}} \int_{T}\left(\tilde{I}_{0} w_{, t}+\tilde{I}_{i} \boldsymbol{w}_{. x_{2}}\right) \cdot \boldsymbol{\tau}\left(\tilde{I}_{0} v_{. t}+\tilde{I}_{i} v_{. x_{2}}\right) d x d t \\
& B(\boldsymbol{v} \cdot \boldsymbol{w})_{\mathrm{BC}}=\int_{I^{2}} \int_{\Gamma} \boldsymbol{w} \cdot \frac{1}{2}\left(\boldsymbol{f}(\tilde{\boldsymbol{g}} ; \boldsymbol{n})-\boldsymbol{f}(\boldsymbol{v} ; \boldsymbol{n})-\boldsymbol{h}^{d}(\boldsymbol{v} \cdot \overline{\boldsymbol{g}}: \boldsymbol{n})\right) d x d t
\end{aligned}
$$

where $h^{d}$ denotes the flux dissipation term incorporated into the total numerical flux.

Theorem 2.5. Global Entropy Norm Stability (Nonlinear Hyperbolic System). The variational formulation (2.3) for nonlinear systems of conservation laws with convex entropy extension and symmetric mean-value flux dissipation

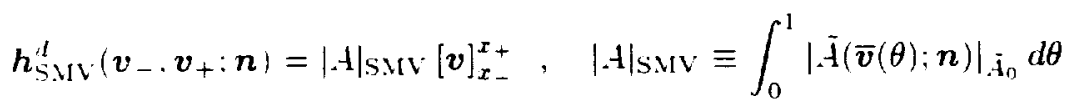

is entropy norm stable (modulo data $\dot{g}$ ) with the following global balance:

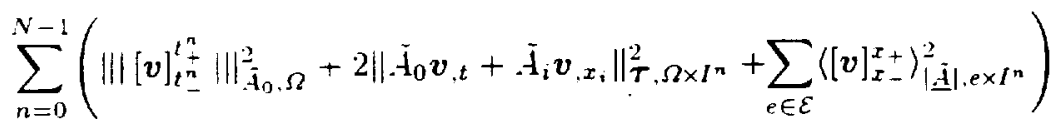

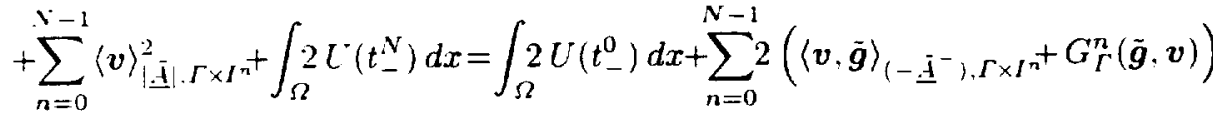

with

$$
|\underline{\underline{I}}(n)|=\int_{0}^{1} 2(1-\theta)\left(\tilde{A}^{+}(\bar{v}(\theta) ; n)_{\bar{A}_{0}}-\tilde{A}^{-}(\bar{v}(1-\theta) ; n)_{\tilde{A}_{0}}\right) d \theta
$$

and

$$
G_{\Gamma}^{n}(\overline{\boldsymbol{g}}, \boldsymbol{v})=\int_{I^{n}} \int_{\Gamma}\left(F(\tilde{\boldsymbol{g}} ; \boldsymbol{n})-\int_{0}^{1} \theta \overline{\boldsymbol{g}} \cdot A(\overline{\boldsymbol{v}}(\theta) ; \boldsymbol{n}) \overline{\boldsymbol{g}} d \theta\right) d x d t
$$

Proof. Construct the energy balance for the interval $\left[t_{-}^{0}, t_{-}^{N}\right]=\cup_{n=0}^{N-1} I^{n}$ by setting $\boldsymbol{w}=\boldsymbol{v}$ and evaluating the various integrals. Consider the time derivative integral

$$
\int_{S^{2}} \int_{I^{n}} v^{T} u_{, t} d t d x=\int_{\Omega} \int_{I^{n}} U_{. t} d t d x=\int_{\Omega}\left(\left[C^{v}\right]_{t_{-}^{n}}^{t_{n}^{n+1}}-\left[C^{C}\right]_{t^{n}}^{t_{+}^{n}}\right) d x
$$


and combine with the jump integral across time slabs. From Corollary 2.3

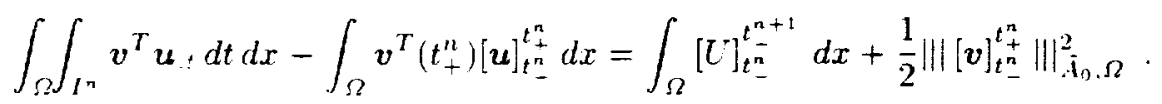

When summed over all time slabs, the first term on the right-hand-side of this equation vanishes except for initial and final time slab contributions. Next, consider the spatial operator term and apply the divergence theorem

$$
\begin{aligned}
\int_{I^{n}} \int_{\Omega} v^{T} f_{x_{i}}^{i} d x d t & =\int_{I^{n}} \int_{\Omega} F_{x_{1}}^{i} d x d t \\
& =\int_{I^{n}} \sum_{e \in \mathcal{E}} \int_{e}-[F(\boldsymbol{v} ; \boldsymbol{n})]_{x_{-}}^{x_{+}} d x d t+\int_{I} \int_{\Gamma} F(\boldsymbol{v} ; \boldsymbol{n}) d x d t
\end{aligned}
$$

where $F(v: n)=n: F^{i}(v)$. From Corollary 2.4 and the definition of $|\underline{1}|$, it follows that

$$
\begin{array}{r}
\left.\int_{I^{n}} \int_{\Omega} v^{T} f_{I_{1}}^{\prime} d x d t-\int_{I^{n}} \sum_{e \in \mathcal{E}} \int_{e}(\langle\boldsymbol{v}\rangle\rangle_{x_{-}}^{x_{+}} \cdot[\boldsymbol{f}(\boldsymbol{n})]_{x_{-}}^{x_{+}}+\frac{1}{2}[\boldsymbol{v}]_{x_{-}}^{x_{+}} \cdot \boldsymbol{h}_{\mathrm{SMV}}^{d}\right) d x d t \\
=\sum_{e \in \mathcal{E}} \frac{1}{2}\left\langle[\boldsymbol{v}]_{x_{-}}^{x_{+}}\right\rangle_{|\underline{\underline{\mathbb{A}}}|, e \times I^{n}}^{2}+\int_{I^{n}} \int_{\Gamma} F(\boldsymbol{v} ; \boldsymbol{n}) d x d t
\end{array}
$$

In summary: collecting terms we have

$$
\begin{aligned}
& B(\boldsymbol{v}, \boldsymbol{v})_{G, \mathrm{LL}}=\int_{\Omega}\left[\mathcal{C}_{t_{-}^{r}}^{t_{-}^{n+1}} d x+\left.\frac{1}{2}\left\|[\boldsymbol{v}]_{t_{-}^{n}}^{t_{+}^{n}}\right\|\right|_{\tilde{A}_{0}, \Omega} ^{2}+\sum_{e \in \mathcal{E}} \frac{1}{2}\left\langle[\boldsymbol{v}]_{x_{-}}^{x_{+}}\right\rangle_{|\underline{\tilde{X}}|, \operatorname{ex} I^{n}}^{2}\right. \\
& -\int_{I^{n}} \int_{\Gamma} F(v ; \boldsymbol{n}) d x d t \text {. }
\end{aligned}
$$

The least-squares integral produces a pure quadratic form without modification

$$
B(v, v)_{\mathrm{LS}}=\left\|\bar{A}_{0} v_{, t}+\bar{A}_{i} v_{, x_{i}}\right\|_{\tau, \Omega \times I^{n}}^{2} .
$$

Finally, consider the boundary condition terms and apply Corollary 2.4

$$
\begin{aligned}
B(\boldsymbol{v}, \boldsymbol{v})_{\mathrm{BC}}= & \int_{I^{n}} \int_{\Gamma}\left(\frac{1}{2} \boldsymbol{v} \cdot(\boldsymbol{f}(\tilde{\boldsymbol{g}} ; \boldsymbol{n})-\boldsymbol{f}(\boldsymbol{v} ; \boldsymbol{n}))-\frac{1}{2} \boldsymbol{v} \cdot|A(\boldsymbol{n})|_{\mathrm{SMV}}(\tilde{\boldsymbol{g}}-\boldsymbol{v})\right) d x d t \\
= & \int_{I^{n}} \int_{\Gamma}\left(F(\tilde{\boldsymbol{g}} ; \boldsymbol{n})-F(\boldsymbol{v} ; \boldsymbol{n})+\frac{1}{2} \int_{0}^{1}(1-2 \theta)(\tilde{\boldsymbol{g}}-\boldsymbol{v}) \cdot \tilde{\mathrm{I}}(\overline{\boldsymbol{v}}(\theta) ; \boldsymbol{n})(\tilde{\boldsymbol{g}}-\boldsymbol{v}) d \theta\right. \\
& \left.-\frac{1}{2} \boldsymbol{g} \cdot(\boldsymbol{f}(\tilde{\boldsymbol{g}} ; \boldsymbol{n})-\boldsymbol{f}(\boldsymbol{v} ; \boldsymbol{n}))-\frac{1}{2} \boldsymbol{v} \cdot|A(\boldsymbol{n})|_{\mathrm{SMV}}(\tilde{\boldsymbol{g}}-\boldsymbol{v})\right) d x d t \\
= & \int_{I^{n}} \int_{\Gamma}\left(F(\tilde{\boldsymbol{g}} ; \boldsymbol{n})-F(\boldsymbol{v} ; \boldsymbol{n})-\int_{0}^{1} \theta \tilde{\boldsymbol{g}} \cdot \tilde{I}(\bar{v}(\boldsymbol{\theta})) \tilde{\boldsymbol{g}} d \boldsymbol{\theta}\right. \\
& \left.\quad+\frac{1}{2} \boldsymbol{v} \cdot \underline{\underline{I}}(\boldsymbol{n}) \mid \boldsymbol{v}-\boldsymbol{v} \cdot \underline{\tilde{I}}^{-}(\boldsymbol{n}) \tilde{\boldsymbol{g}}\right) d x d t .
\end{aligned}
$$

Combining the above results, summing over time slabs, and multiplication by two yields an entropy norm balance equation which bounds the global entropy norm of the system at the final time $T$ in terms of the initial data and boundary data $\bar{g}$. 
Remark 2.6. Yote that when the $\bar{A}_{i}$ matrices are assumed constant, $f^{i}=$ $\tilde{I}_{i} v$ and $F^{\prime}=\frac{1}{2} v \cdot \tilde{I}_{i} v$ so that the additional term $G_{\Gamma}^{n}(\bar{g} \cdot v ; n)$ vanishes identically and a one-to-one correspondence of terms between theorem 2.1 and theorem 2.5 is arhieved.

\subsection{A Simplified DG Method in Symmetric Form}

DG Flux Formulas. Simplification of the discontinuous Galerkin method follows by choosing the discrete symmetric mean-value flux function proposed earlier. i.e.

$\left.h_{\mathrm{D}: \mathrm{MV}}\left(\boldsymbol{v}_{-} \cdot v_{+}: \boldsymbol{n}\right)=\frac{1}{2}\left(\boldsymbol{f}\left(\boldsymbol{v}_{-} ; \boldsymbol{n}\right)+\boldsymbol{f}\left(\boldsymbol{v}_{+} ; \boldsymbol{n}\right)\right)-\frac{1}{2} \sum_{q=1}^{N} u_{q} \mid \cdot \bar{I}\left(\overline{\boldsymbol{v}}\left(\theta_{q}\right)\right) ; \boldsymbol{n}\right)\left.\right|_{\dot{A}_{0}}[\boldsymbol{v}]_{x_{-}}^{x_{+}}$

with $|\bar{A}|_{.}=\bar{R} \mid .1 \dot{R}^{T} \cdot \bar{v}(\theta)=v\left(x_{-}\right)+\theta[v]_{x_{-}}^{x_{+}}$. By using sufficient order numerical quadrature and subdivision of the state-space path integration at points of non-differentiability, the $\boldsymbol{h}_{\mathrm{DS}}^{d}$. flux can be made arbitrarily close to $h_{S M V}^{d}$ for which noniinear stability in the DG method follows from the analysis of Sect. 2.2. Suppose that elements of $\left.\mid \bar{A}\left(\overline{\boldsymbol{v}}\left(\theta_{q}\right)\right) ; \boldsymbol{n}\right)\left.\right|_{\overline{\boldsymbol{A}}_{0}}$ remain bounded for $\theta \in[0.1]$ independent of $N$. Using $N$ point Gaussian quadrature

$$
{ }_{1} \boldsymbol{h}_{\mathrm{DSMV}}^{d}-\boldsymbol{h}_{\mathrm{SMV}}^{d} \|_{2}=O\left([\boldsymbol{v}]^{2 N+1}\right) \text {. }
$$

Next. we consider single-point quadrature formulas.

Theorem 2.7. Discrete Symmetric Mean-Value Flux. Let $v$. be a state such that

$$
[\boldsymbol{v}]_{x_{-}}^{x_{-}} \cdot|\tilde{\mathcal{A}}(\boldsymbol{v}: n)|[\boldsymbol{v}]_{x_{-}}^{x_{+}}=\sup _{0 \leq \theta \leq 1}[\boldsymbol{v}]_{x_{-}}^{x_{+}} \cdot|\tilde{\mathcal{A}}(\overline{\boldsymbol{v}}(\theta) ; \boldsymbol{n})|_{\tilde{A}_{0}}[\boldsymbol{v}]_{x_{-}}^{x_{+}}
$$

The variational formulation (2.3) with numerical flux function

$$
\left.h_{\mathrm{DSMV}}\left(v_{-}, v_{+}: n\right)=\frac{1}{2}\left(f\left(v_{-} ; n\right)+f\left(v_{+}: n\right)\right)-\frac{1}{2} \mid \tilde{A}\left(v_{*}\right) ; n\right)\left.\right|_{\tilde{A}_{0}}[v]_{x_{-}}^{x_{+}}
$$

is energy bounded in the sense of Theorem 2.5.

Proof. It is sufficient to show that the given flux dissipation

$$
h_{\mathrm{DSMV} *}^{d}=\left|\tilde{A}\left(v_{*} ; n\right)\right|_{\tilde{A}_{0}}[\boldsymbol{v}]_{x_{-}}^{x_{+}}
$$

exceeds the symmetric mean-value value flux dissipation. This is reflected by the algebraic condition

$$
[v]_{x_{-}}^{x_{+}} \cdot h_{\mathrm{SMV}}^{d} \leq[v]_{x_{-}}^{x_{+}} \cdot h_{\mathrm{DSMV} *}^{d} .
$$

From the symmetric mean-value flux definition

$$
\begin{aligned}
{[\boldsymbol{v}]_{x_{-}}^{x_{+}} \cdot h_{\text {SMV }}^{d} } & =[v]_{x_{-}}^{x_{+}} \cdot \int_{0}^{1}|\tilde{I}(\bar{v}(\theta) ; n)|_{\tilde{A}_{1}} d \theta[v]_{x_{-}}^{x_{+}} \\
& \leq \sup _{0 \leq \theta \leq 1}[v]_{x_{-}}^{x_{+}} \cdot|\overline{\mathcal{I}}(\overline{\boldsymbol{v}}(\theta) ; n)|_{\bar{A}_{1}}[\boldsymbol{v}]_{x_{-}}^{x_{+}}
\end{aligned}
$$




$$
\begin{aligned}
& =[\boldsymbol{v}]_{x-}^{x_{+}} \cdot\left|\overline{\mathcal{A}}\left(v_{*} ; n\right)\right|_{\bar{A}_{0}}[\boldsymbol{v}]_{x_{-}}^{\boldsymbol{x}_{+}} \\
& =[\boldsymbol{v}]_{x_{-}}^{x_{+}} \cdot \boldsymbol{h}_{\mathrm{DSNMV}}^{d} .
\end{aligned}
$$

This establishes nonlinear stability of the DG method using the simplified flux function.

Remark 2.8. Unfor 1 nately, the state $v_{*}$ is not generally known in closed form. Cockburn and Shu [ 4$]$ have shown impressive results using the simpler Lax-Friedrichs flux. It is straightforward to derive a corresponding "symmetric Lax-Friedrichs" numerical flux function

$$
h_{\mathrm{SLF}}\left(v_{-} \cdot v_{+}: n=\frac{1}{2}\left(f\left(v_{-} ; n\right)+f\left(v_{+} ; n\right)\right)-\frac{1}{2} \lambda_{\max } \tilde{I}_{0}\left(v_{*}\right)[v]_{x_{-}}^{x_{+}}\right.
$$

with $\lambda_{\max }=\sup _{0 \leq m \leq 1}\left(\max _{1 \leq i \leq m}\left(\Lambda_{i i}(\bar{v}(\theta))\right)\right)$. Nonlinear entropy norm stability follows starting from Theorem $2 . \bar{T}$

$$
\begin{aligned}
& { }_{\{} v_{x_{-}}^{x_{-}} \cdot h_{S M V}^{d} \leq\left[\boldsymbol{v}_{x_{-}}^{z_{-}} \cdot\left|\overline{\mathcal{A}}\left(v_{*} ; n\right)\right|_{\tilde{A}_{0}}[\boldsymbol{v}]_{x_{-}}^{x_{+}}\right. \\
& =\left[v_{z_{-}}^{z_{-}} \cdot \dot{R}\left(v_{*} ; n\right)\left|\cdot 1\left(v_{*} ; n\right)\right| \tilde{R}^{T}\left(v_{*} ; n\right)[v]_{x_{-}}^{x_{+}}\right. \\
& \leq \sup _{0 \leq \dot{0} \leq 1}\left(\max _{1 \leq i \leq m}\left(\Lambda_{i i}(\bar{v}(\theta))\right)\right)[v]_{x_{-}}^{x_{+}} \cdot \tilde{R}\left(v_{*} ; n\right) \tilde{R}^{T}\left(v_{*} ; n\right)[v]_{x_{-}}^{x_{+}} \\
& =\lambda_{\max }[\boldsymbol{v}]_{x_{-}}^{x_{+}} \cdot \tilde{A}_{0}\left(\boldsymbol{v}_{*} ; \boldsymbol{n}\right)[\boldsymbol{v}]_{x_{-}}^{x_{+}} \text {. }
\end{aligned}
$$

Finally, for systems such as the Euler equations of gas dynamics that exhibit the propert $y \frac{\partial^{4} u}{\partial v_{i} v_{j} v_{i} v_{l}} z_{i} z_{j} z_{k} z_{l}>0,|z| \neq 0$, we have

$[v]_{x_{-}}^{x_{-}} \cdot \bar{A}_{0}(v: n)[\boldsymbol{v}]_{I_{-}}^{x_{-}} \leq \max \left([\boldsymbol{v}]_{x_{-}}^{x_{+}} \cdot \bar{A}_{0}(\overline{\boldsymbol{v}}(0) ; \boldsymbol{n})[\boldsymbol{v}]_{x_{-}}^{x_{+}},[\boldsymbol{v}]_{x_{-}}^{x_{+}} \cdot \bar{A}_{0}(\overline{\boldsymbol{v}}(1) ; \boldsymbol{n})[\boldsymbol{v}]_{x_{-}}^{x_{+}}\right)$

thereby avoiding the need for calculating $v_{*}$ altogether, see [1] for details.

DG Jacobian Derivatives. Using the discrete mean-value fluxes, it becomes straightforward to compute Jacobian derivatives of various terms. For example. to compute derivatives of $|-\bar{A}|_{\vec{A}_{0}}$ with respect to a vector $\boldsymbol{w}$, chain-rule differentiation is used

$$
\frac{\partial|\overline{\mathcal{I}}(\boldsymbol{n})|_{\overline{\mathcal{A}}_{0}}}{\partial \boldsymbol{w}}=\frac{\partial \tilde{R}(\boldsymbol{n})}{\partial \boldsymbol{w}} \cdot \mathbf{l}(\boldsymbol{n})\left|\tilde{R}^{T}(\boldsymbol{n})+\tilde{R}(\boldsymbol{n}) \frac{\partial|\lambda(\boldsymbol{n})|}{\partial \boldsymbol{w}} \bar{R}^{T}(\boldsymbol{n})+\tilde{R}(\boldsymbol{n})\right| .1(n) \mid \frac{\partial \bar{R}^{T}(\boldsymbol{n})}{\partial \boldsymbol{w}}
$$

Note that a high degree of computational efficiency can be achieved in the calculation of these Jacobian terms by exploiting the transpose symmetry of. intermediate products.

\subsection{Simplified Least-Squares Stabilization in Symmetric Form}

Consider an isoparametric element mapping $\boldsymbol{\xi} \mapsto \mathbf{x}$ from a unit element space $\boldsymbol{\xi}$ to a physical space $\mathbf{x}$. In the papers by Hughes and Mallet [7] and Shakib [10]. they proposed the following form for $\tau$ appearing in (2.3) on a mapped element

$$
\tau_{p}=|\bar{B}|_{p . i_{0}}^{-1}, \quad|\bar{B}|_{p . \overline{\mathrm{I}}_{0}}=\left(\sum_{i=0}^{d}\left|B^{i}\right|^{p}\right)^{1 / p} \quad \overline{\mathrm{I}}_{0}, \quad B^{i}=\sum_{j=0}^{d} \xi_{, x_{j}}, \mathcal{A}_{j} .
$$


Equation 2.20$)$ is of the same form given earlier in (1.15). In standard implementations of least-squares stabilization, $p=2$ is used. In light of the Eigenvector Scaling Theorem 1.1. it is useful to revisit the derivation of $\tau$ with $p=1$. Let $\bar{B}^{2}=B^{2}$. $\tilde{\mathrm{t}}_{0}$, from $(1.16)$ it follows that

$$
\left.\tau_{1}=|\tilde{B}|_{1 . . \bar{i}}^{-1}=\left[\left|\nabla \xi^{0}\right| \tilde{t}_{0}+\sum_{i=1}^{l}\left|\nabla \xi^{i}\right| \tilde{R}\left(\boldsymbol{n}^{i}\right)\left|.1\left(\boldsymbol{n}^{i}\right)\right| \tilde{R}^{T} \boldsymbol{n}^{i}\right)\right]^{-1}
$$

using the entropy scaled eigenvectors $\tilde{R}\left(n^{i}\right)$ of $\tilde{B}^{i}$. This represents a substantial simplification of the $\tau$ matrix calculation.

\section{Concluding Remarks}

Simplified forms of the DG. DGLS, and GLS schemes have been presented and analyzed for first-order systems of conservation laws with convex entropy extension. Vinmerical examples are given in [1] using linear. quadratic, and cubic element approximation.

\section{References}

1. T. J. Barth. Simplified discontinuous Galerkin methods for svstems of conservation laws with convex extension. Math. Comp., submitted 1999.

2. T.J. Barth. Numerical methods for gasdynamic systems on unstructured meshes. In Kröner. Ohlberger. and Rohde, editors, An Introduction to Recent Detelopments in Theory and Numerics for Conservation Laws, volume 5 of Lecture Notes in Computational Science and Engineering. pages 195-285. Springer-Verlag, Heidelberg, 1998.

3. B. Cockburn, S. Hou, and C.W. Shu. TVB Runge-Kutta local projection discontinuous Galerkin finite element method for conservation laws IV: The multidimensional case. Math. Comp., 54:545-581, 1990.

4. B. Cockburn and C.W. Shu. The Runge-Kutta discontinuous Galerkin method for conservation laws V: Multidimensional systems. Technical Report 201737, ICASE. NASA Langley R.C., 1997.

5. K. O. Friedrichs and P. D. Lax. Systems of conservation laws with convex extension. Proc. Vat. Acad. Sci. USA, 68(8):1686-1688, 1971.

6. F. R. Gantmacher. Matrix Theory. Chelsea Publishing Company, New York, N.Y., 1959.

7. T. J. R. Hughes and M. Mallet. A new finite element formulation for CFD: III. the generalized streamline operator for multidimensional advective-diffusive systems. Comp. Weth. Appl. Mech. Engrg., 58:305-328, 1986.

8. C. Johnson and J. Pitkäranta. An analysis of the discontinuous Galerkin method for a scalar hyperbolic equation. Math. Comp., 46:1-26, 1986.

9. .I. S. Mlock. Systems of conservation laws of mixed type. J. Diff. Eqns., 37:70-88. 1980.

10. F. Shakib. Finite Element Analysis of the Compressible Euler and NavierStokes Equations. PhD thesis, Stanford University, Departinent of Mechanical Engineering, 1988 . 
